\title{
Evaluation of technician supervised treadmill exercise testing in a cardiac chest pain clinic
}

\author{
G Davis, S Ortloff, A Reed, G Worthington, D Roberts
}

\begin{abstract}
Objective-To determine the efficacy and safety of trained cardiac technicians independently performing treadmill exercise stress tests as part of the assessment of patients with suspected angina pectoris. Design-Retrospective comparison of 250 exercise tests performed by cardiac technicians and 225 tests performed by experienced cardiology clinical assistants (general practitioners who perform regular NHS cardiology duties), and consultant cardiologists over the same time period.

Setting-Regional cardiac centre with a dedicated cardiac chest pain clinic.

Patients-All patients were referred by their general practitioners with a history of recent onset of chest pain, which was suspected to be angina pectoris.

Outcome measures-Peak workload achieved, symptoms, indications for termination, complications.

Results-The diagnostic yield of technician supervised tests (percentage positive or negative) was similar to that of medically supervised tests $(76 \% v 69 \%$, NS). The average peak workload achieved by patients was less by 1.2 mets $(p<0.005)$. This was probably due to more tests being terminated earlier due to chest pain and ST segment depression in the technician group compared with doctors $(10 \%$ and $16 \% v 5 \%$ and $11 \%$ respectively, $p=0.06$ and 0.07$)$. One patient in the technician supervised group developed a supraventricular tachycardia during the recovery phase of the exercise test.

Conclusions-Technician supervised stress testing is associated with a high diagnostic rate and low complication rate in patients with suspected ischaemic heart disease. Its efficacy is comparable to tests supervised by experienced doctors and its use should be encouraged.

(Heart 1998;79:613-615)
\end{abstract}

Keywords: exercise stress testing; cardiac technician; angina pectoris; audit

Regional Cardiac Centre, Victoria Hospital, Blackpool, UK

Correspondence to: Dr Davis, Regional Cardiac Centre, Victoria Hospital, Blackpool FY3 8NR, UK.

Accepted for publication 5 September 1997
Angina pectoris is a common symptom with an estimated incidence for the United Kingdom of approximately 22000 new cases per year. ${ }^{1}$ The majority of new cases present to general practitioners who subsequently refer up to $25 \%$ to a hospital physician. ${ }^{2}$ It has been shown that new patients presenting with angina in the community do not have a benign prognosis; there is evidence that both men and women have a threefold increased risk of developing unstable angina, myocardial infarction, or death within two years of first presentation. ${ }^{3}$ The 1993 recommendation of the Royal College of Physicians and the British Cardiac Society is that newly diagnosed cases of angina in patients under 70 years of age should have cardiological referral and, if appropriate, exercise stress testing. This would involve an estimated 3000 exercise tests per million population per year. ${ }^{4}$ At present, exercise tests are mainly supervised by junior doctors with varying levels of cardiological experience, which may not be appropriate for this service commitment. In addition, the effect of the Calman changes on cardiology training and the Royal College of Physicians core training syllabus for general medical senior house officer training would decrease junior doctors' availability for supervising exercise stress testing. One way of meeting the increased demand is by the routine use of technician supervised exercise stress testing in low risk populations. This can be performed in accordance with the 1993 guidelines of the Medical Practice Committee and Council of the British Cardiac Society. ${ }^{5}$ We evaluated our experience with this form of stress testing supervision as an aid to rapid patient diagnosis and management in a cardiac chest pain clinic. The efficacy and complication rates were compared with those of exercise tests performed by cardiology clinical assistants and consultant cardiologists.

\section{Methods}

PATIENT POPULATION

The study population comprised 250 patients who were referred by their general practitioner with a new diagnosis of possible angina pectoris. There was no previous history of ischaemic heart disease, and patients were clinically assessed by a cardiology registrar before exercise testing to ensure that angina was the possible diagnosis and that the patient was suitable for exercise testing using a treadmill. All patients with proven ischaemic heart disease, unstable symptoms, or other illnesses were excluded. A detailed request form was completed and signed by the cardiology registrar before the technician performed the test. This provided information about the type of treadmill test required, the resting ECG and blood pressure, and cardiac drugs. It also confirmed that the patient was examined and that there were no features of aortic stenosis, acute myocardial infarction, or other contraindications. The results of 205 patients exercised by clinical assistants and 20 by consultant cardiologists were analysed for comparison. 
PROTOCOL

Technician only tests were performed by three technicians who were of senior grade (medical technical officer 3 or above) and were trained in basic resuscitation and advanced cardiac life support. They had each been performing medically supervised exercise stress testing for an average of 15 years and had attended meetings on cardiac stress testing, including those organised by the Society of Cardiological Technicians. Technicians were supervised by local cardiology consultants and experienced physicians for approximately 50 tests before going solo. Patients were supervised by one of the three technicians for each test, with another technician trained in basic life support also present. Two experienced clinical assistants and three consultant cardiologists performed medically supervised tests. A cardiac technician assisted all medical personnel.

All exercise tests were performed in a dedicated exercise room in close proximity to the cardiac ward. There was a cardiac arrest call alarm and standard resuscitation equipment.

In our unit all patients give written consent before exercise stress testing. Patient consent was obtained by the technician, who was also responsible for blood pressure measurements and terminating the test. Before starting the test the technician also completed a preexercise checklist. This consisted of confirmation that all necessary checks have been performed (for example, notes present, blood pressure satisfactory, request form fully completed and signed, no new ECG changes) and that all items on the resuscitation trolley and safety equipment had been checked before the session. At the end of the test the patient returned to the outpatient department with the technician's report on the exercise test and a final consultation with the referring doctor.

\section{END POINTS}

The results from the tests performed by technicians were compared retrospectively with tests performed by the clinical assistants and consultant cardiologists over the same period. Only exercise tests performed by doctors for a similar indication (diagnosis of chest pain presumed to be angina pectoris) were included in the analysis. End points analysed were the type and duration of the exercise

Table 1 Clinical details of the patients

\begin{tabular}{|c|c|c|c|}
\hline & $\begin{array}{l}\text { Technician } \\
\text { only }\end{array}$ & $\begin{array}{l}\text { Medically } \\
\text { supervised }\end{array}$ & $p$ value \\
\hline Number of patients & 250 & 225 & \\
\hline Male/female (\%) & $56 / 44$ & $52 / 48$ & \\
\hline Mean (SD) age (years) & $56(10)$ & $56(10)$ & \\
\hline Mean (SD) work load (mets) & $7.2(3.5)$ & $8.4(3.6)$ & $<0.005$ \\
\hline \multicolumn{4}{|l|}{ Indications for termination } \\
\hline Maximum heart rate & $60(24 \%)$ & $56(25 \%)$ & NS \\
\hline Shortness of breath & $42(17 \%)$ & $45(20 \%)$ & NS \\
\hline Chest pain & $25(10 \%)$ & $12(5 \%)$ & 0.06 \\
\hline ST depression $>0.2 \mathrm{mV}$ & $41(16 \%)$ & $24(11 \%)$ & 0.07 \\
\hline Blood pressure changes $\star$ & $18(7 \%)$ & $2(<1 \%)$ & $<0.005$ \\
\hline Non-specific symptomst & $64(25 \%)$ & $86(38 \%)$ & $<0.005$ \\
\hline
\end{tabular}

^Hypertension, hypotension or inability to obtain a blood pressure recording; ffatigue or musculoskeletal pain.

Mets, metabolic equivalents of oxygen consumption. test, peak patient workload achieved, symptoms experienced, and indications for termination of the test. A test was regarded as positive if the ST segment response was horizontal or showed a down sloping depression of more than $0.1 \mathrm{mV}$ at $80 \mathrm{~ms}$ after the J point. A negative test was recorded if the pulse and blood pressure responses were normal and no ECG morphological or rhythm changes were present, despite attaining $80 \%$ of the target heart rate. Tests not fitting either criterion were regarded as inconclusive.

\section{STATISTICAL ANALYSIS}

All data are expressed as mean (SD). Statistical significance was assessed by the unpaired Student's $t$ test for continuous variables and the $\chi^{2}$ test for categorical variables; $p<0.05$ was considered significant.

\section{Results}

Technicians supervise approximately $53 \%$ of all exercise tests performed on patients referred with suspected angina pectoris. However, they exercise all patients who attend the chest pain clinic and who require an exercise test. The age and sex distribution of both patient groups were similar (table 1). Of the 250 tests performed by three technicians, a diagnostic result (positive or negative) was obtained in $76 \%$. One patient developed a supraventricular tachycardia during the recovery phase. Doctors achieved similar diagnostic results $(69 \%, \mathrm{NS})$. The average workload for patients exercised by technicians was lower than patients exercised by doctors (7.2 (3.5) mets $v 8.4$ (3.6) mets, $\mathrm{p}<0.005)$. Tests were terminated in both groups in $24 \%$ of patients who achieved their maximum predicted heart rate, while the indication for termination was shortness of breath in $17 \%$ of technician only tests compared with $20 \%$ in the medically supervised group. A higher percentage of patients was stopped because of chest pain and the development of ST segment depression in the technician group (10\% and $17 \%$ respectively) compared to the physician group (5\% and $10 \%$ respectively; $\mathrm{p}=0.06$ and 0.07 ).

\section{Discussion}

It is still the practice in most district general hospitals and cardiac units for exercise tests to be supervised in general by senior house officers with little cardiological experience. Our results show that appropriately trained cardiac technicians can supervise low risk tests and obtain results similar to experienced medical personnel. There are no definitive answers on which grade of technician is appropriate. The BCS guidelines (a fully trained technician of senior grade) have been interpreted by the Society of Cardiological Technicians ${ }^{6}$ as being an MTO 3 and above. Our NHS trust has indemnified named technicians against any liability that may arise from technician only stress testing performed in accordance with the guidelines described and in-house protocols. The role of the assistant to the trained technician is to help in physically moving a patient if this becomes necessary. 
The peak workload in patients exercised by technicians was less than in the physician supervised group. This is probably because more patients were stopped earlier by chest pain, ST segment depression, and blood pressure response than in the physician group. Patients exercised by physicians had fewer responses of this type and were thus more likely to have a test terminated as a result of non-specific symptoms (table 1).

The safeguards suggested in the British Cardiac Society/Medical Practice Committee guidelines $^{5}$ and the directions given by the stress working group of the Society of Cardiological Technicians ${ }^{6}$ will help to maintain the low complication rate of the procedure. It is well known that observational factors recorded during the exercise test, such as the development of symptoms, blood pressure responses, and ECG changes persisting into recovery, aid in the ability to arrive at a diagnosis of ischaemic heart disease. ${ }^{7}$ It is our concern that not only must safety be ensured but the usefulness of the test must also be maintained by adequate supervision and by obtaining as much information as possible. The training of cardiac technicians for this role needs to be structured. It should include an awareness of basic exercise physiology and the various cardiac and ECG responses to exercise. An essential part of maintaining standards would involve continuing education in the form of reviewing results and interpretation of data with experienced physicians, and discussion of management decisions that may have arisen in particular cases.

At Blackpool, technicians independently perform and report $30 \%$ of all exercise tests. Stress testing requires clinical input in the screening of referred patients and the interpretation of the test results if it is to be a useful investigation in the diagnosis of chest pain in patients referred by their general practitioner. $^{8}$ The use of technician only services with clinical support would deal with the number of investigations required to provide an adequate standard of cardiovascular care. This is particularly important in the light of the current changes in the structure of medical training, which would necessarily affect medical staff service commitment.

We suggest that adequately trained technicians would allow the exercise ECG to remain a valuable and available investigation, aiding clinicians in determining prognosis and the need for invasive cardiac investigation.

We thank the technicians and clinical assistants for performing the exercise tests and consultant cardiologists for use of their patients.

1 Gandhi MM, Lampe FC, Wood DA. Incidence, clinical characteristics and short term prognosis of angina pectoris. Br Heart F 1995;73:193-8

2 Gandhi MM, Lampe FC, Wood DA. Management of angina pectoris in general practice: a questionnaire survey of general practitioners. Br f Gen Pract 1995;45:11-13.

3 Murabito JM, Anderson KM, Kannel WB, et al. Risk of coronary heart disease in subjects with chest discomfort: the Framingham heart study. Am f Med 1990;89:297-302.

4 De Bono D, Hopkins A, for British Cardiac Society and Royal College of Physicians joint working party. The investigation and management of stable angina. $\mathcal{f} R$ Coll Physicians Lond 1993;27:267-73.

5 Guidelines for exercise testing when there is not a doctor present. Recommendations of the Medical Practice present. Recommendations of the Medical Practice
Committee and Council of the British Cardiac Society, 1993 (unpublished)

6 Report of the stress working group of the Society of Cardiological Technicians. London: Society of Cardiological Technicians, 1994

7 Mark DB, Shaw L, Harrell FE, et al. Prognostic value of a treadmill exercise score in outpatients with suspected coronary artery disease. N Engl f Med 1991;325:849-53.

8 Sulke AN, Paul VE, Taylor CJ, Roberts RH, Norris ADC. Open access exercise electrocardiography: a service to improve management of ischaemic heart disease by general practitioners. f R Soc Med 1991;84:590-4. 